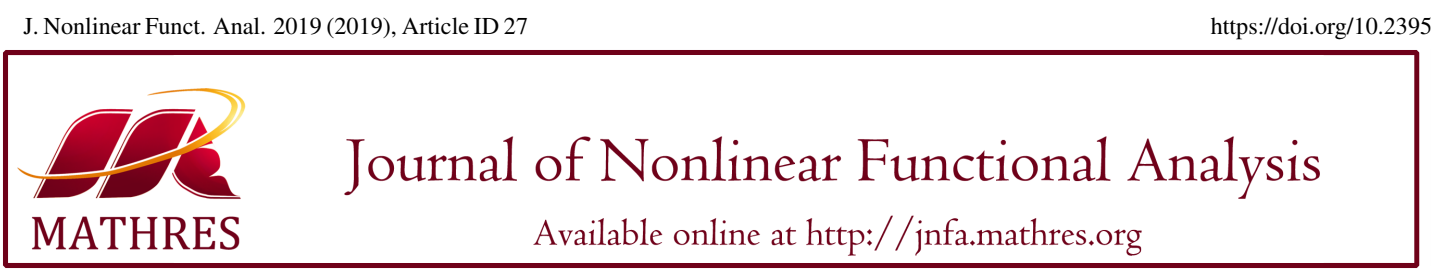

https://doi.org/10.23952/jnfa.2019.27

\title{
VARIATION OF THE FIRST EIGENVALUE OF $p$-LAPLACIAN ON EVOLVING GEOMETRY AND APPLICATIONS
}

\author{
ABIMBOLA ABOLARINWA ${ }^{1, *}$, OLUKAYODE ADEBIMPE $^{1}, \mathrm{JING}^{\text {MAO }}{ }^{2}$ \\ ${ }^{1}$ Department of Physical Sciences, Landmark University, P.M.B. 1001, Omu-Aran, Kwara State, Nigeria \\ ${ }^{2}$ Faculty of Mathematics and Statistics, Key Laboratory of Applied Mathematics of Hubei Province, \\ Hubei University, Wuhan 430062, China
}

\begin{abstract}
Let $(M, g)$ be an $n$-dimensional compact Riemannian manifold whose metric $g(t)$ evolves by the generalised abstract geometric flow. This paper discusses the variation formulas, monotonicity and differentiability for the first eigenvalue of the $p$-Laplacian on $(M, g(t))$. It is shown that the first nonzero eigenvalue is monotonically nondecreasing along the flow under certain geometric conditions and that it is differentiable almost everywhere. These results provide a unified approach to the study of eigenvalue variations and applications under many geometric flows.
\end{abstract}

Keywords. Geometric flows; $p$-Laplacian; Eigenvalues; Monotonicity; Riemannian manifold.

2010 Mathematics Subject Classification. 53C44, 58C40.

\section{INTRODUCTION}

This paper discusses the variation formulas, monotonicity and differentiability for the first eigenvalue of the $p$-Laplacian on an $n$-dimensional closed Riemannian manifold whose metric evolves by a generalised geometric flow.

1.1. Geometric flow. Let $(M, g)$ be an $n$-dimensional closed Riemannian manifold $(n>1)$. Let $g(x, t)$ be a one parameter family of metrics for $t \in[0, T]$ and $x \in M$. We say that $g(x, t)$ is a generalised geometric flow if it evolves by the following equation

$$
\frac{\partial}{\partial t} g(x, t)=-2 h(x, t), \quad(x, t) \in M \times[0, T]
$$

\footnotetext{
${ }^{*}$ Corresponding author.

E-mail addresses: A.Abolarinwa1@gmail.com (A. Abolarinwa), adebimpe.olukayode@1mu.ng.edu (O. Adebimpe), jiner120@163.com (J. Mao).

Received March 7, 2019; Accepted June 24, 2019.

The first version of the paper was completed during the first author's research visit to African Institute for Mathematical Sciences (AIMS), Senegal in February 2016 and has appeared in preprint arxiv.org 1604.05884.
}

(C)2019 Journal of Nonlinear Functional Analysis 
with $g(x, 0)=g_{0}(x)$, where $0<T<T_{\varepsilon}$ is the maximal time of existence, i.e., $T_{\varepsilon}$ is the first time where the flow blows-up and $h$ is a general time-dependent symmetric 2-tensor. Here $h$ is assumed to be smooth in both variables $t$ and $x$. This is obvious since $g$ is also smooth in both variables. The scaling factor 2 in (1.1) is insignificant while the negative sign may be important in some specific applications for the purpose of keeping the flow either forward or backward in time.

Two popular examples of geometric flows in this category are: the Ricci flow with $h$ being the Ricci curvature tensor, and the mean curvature flow with $h=H \Pi$ (where $H$ is the mean curvature and $\Pi$ is the second fundamental form on $M$ ). Other examples include Yamabe flow, Ricci-harmonic flow, RicciBourguignon flow. The appendix of this paper gives details on some examples of geometric flow.

One may impose boundedness condition on tensor $h$. In fact, such boundedness and sign assumptions on $h$ are preserved as long as the flow exists, so it follows that the metrics are uniformly equivalent. Precisely, if $-K_{1} g \leq h \leq K_{2} g$, where $g(t), t \in[0, T]$ is the flow, then

$$
e^{-K_{1} T} g(0) \leq g(t) \leq e^{K_{2} T} g(0) .
$$

To see the last bounds, we consider the evolution of a vector form $|X|_{g}=g(X, X), X \in T_{X} M$. From (1.1) and the boundedness of the tensor $h$, we have $\left|\partial_{t} g(X, X)\right| \leq K_{2} g(X, X)$, which implies (by integrating from $t_{1}$ to $t_{2}$ )

$$
\left|\log \frac{g\left(t_{2}\right)(X, X)}{g\left(t_{1}\right)(X, X)}\right| \leq\left. K_{2} t\right|_{t_{1}} ^{t_{2}}
$$

Taking the exponential of this estimate with $t_{1}=0$ and $t_{2}=T$ yields $|g(t)| \leq e^{k_{2} T} g(0)$ from which the uniform boundedness of the metric follows. Thus, if there holds boundedness assumption

$$
-K_{1} g \leq h \leq K_{2} g,
$$

the metric $g(t)$ are uniformly bounded below and above for all time $0 \leq t \leq T$ under the geometric flow (1.1). Then, it does not matter what metric we use in the argument that follows.

1.2. Eigenvalue of $p$-Laplacian. The $p$-Laplace operator is defined by

$$
\Delta_{p, g} f(x):=\operatorname{div}\left(|\nabla f|^{p-2} \nabla f\right)(x)
$$

for $p \in[1, \infty)$, where div is the divergence operator, and the adjoint of gradient ( $\mathrm{grad}$ ) for the $L^{2}$-norm induced by $g$ on the space of differential forms. When $p=2, \Delta_{2, g}$ is the usual Laplace-Beltrami operator. The eigenvalues and the corresponding eigenfunctions of $\Delta_{p, g}$ satisfy the following nonlinear eigenvalue problem

$$
\Delta_{p, g} f=-\lambda|f|^{p-2} f, \quad f \neq 0 .
$$

It is well known that the principal symbol of (1.2) is nonnegative everywhere and strictly positive at the neighbourhood of the point where $\nabla f \neq 0$. We also know that (1.2) has weak solutions with only partial regularity of class $C^{1, \alpha},(0<\alpha<1)$ in general. Interested readers can find the classical papers by Evans [1] and Tolksdorff [2]. Notice that the least eigenvalue of $\Delta_{p, g}$ on closed manifold is zero with the corresponding eigenfunction being a constant. Hence, we refer to the infimum of the positive eigenvalues as the first nonzero eigenvalue or simply the first eigenvalue. The first eigenvalue of $\Delta_{p, g}$ is characterised by the min-max principle

$$
\lambda_{p, 1}=\inf _{f}\left\{\frac{\int_{M}|\nabla f|_{g}^{p} d \mu_{g}}{\int_{M}|f|_{g}^{p} d \mu_{g}} \mid f \neq 0, f \in W^{1, p}(M)\right\},
$$


satisfying the following constraint $\int_{M}|f|_{g}^{p-2} f d \mu_{g}=0$, where $d \mu_{g}$ is the volume measure on $(M, g)$. Obviously, the infimum does not change when one replaces $W^{1, p}(M)$ by $C^{\infty}(M)$. The corresponding eigenfunction is the energy minimizer of Rayleigh quotient (1.3) and satisfies the following Euler-Lagrange equation

$$
\int_{M}\left[|\nabla f|^{p-2}\langle\nabla f, \nabla \phi\rangle-\lambda|f|^{p-2}\langle f, \phi\rangle\right] d \mu_{g}=0
$$

for $\phi \in C_{0}^{\infty}(M)$ in the sense of distribution.

It is well-known that $p$-Laplacian has discrete eigenvalues but still remains unknown whether it only has discrete eigenvalues for bounded connected domains. Another well-known results tell us that the first nonzero eigenvalue is simple and isolated $[3,4]$. Here the simplicity shows that any nontrivial eigenfunction corresponding to $\lambda_{p, 1}$ does not change sign and that any two first eigenfunctions are constant multiple of each other.

In contrast to the spectrum of the Laplace-Beltrami operator (the case $p=2$ ), the $p$-Laplacian is nonlinear in general. Moreover, it is not known if $\lambda_{p, 1}$ or its corresponding eigenfunction is $C^{1}$-differentiable (or even locally Lipschitz) along any geometric flow of the form (1.1). However, it has been pointed out that the differentiability for the case $p=2$ is a consequence of eigenvalue perturbation theory; see, for instance, [5, 6] and the references therein. For this reason, any approach (e.g. [7]) that assumes differentiability of eigenvalues and eigenfunctions under the flow can only be applied to the case $p=2$. Now to avoid the differentiability assumption on the first eigenvalue and the corresponding eigenfunction in the case $p \neq 2$, we shall apply techniques introduced in [8] (also used in $[9,10,11]$ ) under the Ricci flow to study the variation and monotonicity of $\lambda_{p, 1}(t)=\lambda_{p, 1}(t, f(t))$, where $\lambda_{p, 1}(t, f(t))$ and $f(t)$ are assumed to be smooth. The evolution and the monotonicity formulas for the first eigenvalue derived here do not depend on the evolution of the eigenfunction. The eigenfunction only needs to satisfy certain normalisation condition.

There are many results on the evolution and monotonicity of eigenvalues of the Laplace operator on evolving manifolds with or without curvature assumptions. One can find [8, 9, 12, 13, 14] under the Ricci flow, $[9,15,16,17]$ under Ricci-harmonic flow and [18] along abstract geometric flow with entropy methods. The study of the properties of eigenvalues of the $p$-Laplacian on evolving manifold is still very young. The main aim of this paper is to investigate if those known properties of $\lambda_{p, 1}$ on static metric and for the case $p=2$ on evolving metric can be extended to various geometric flows. We however intend to develop a unified algorithm that can be used for this purpose on time-dependent metrics. Many interesting results concerning the behaviour of $\lambda_{p, 1}$ can be found in $[3,4,19]$ for static metrics and $[9,11,15,16,20,21,22]$ for evolving metrics along various geometric flow.

The rest of this paper is planned as follows. Section 2 firstly gives some notations about differential geometry and analytic tools used in the paper and then state the main results of the paper. We also state (without proofs) some technical lemmas about variations of some geometric objects relating to the eigenvalues under the flow (1.1). Section 3 discusses the proofs of the main results of the paper. Finally, a comprehensive Appendix is added. Here, some examples of geometric flows where the approaches used in this paper are applicable are listed. In fact, this section reveals that our generalised geometric flow is not a trivial generalisation. 


\section{PREliminaries AND MAin Results}

2.1. Notation. Throughout this paper, $M$ will be taken to be a closed manifold (i.e., compact without boundary). Most of our calculations are done in local coordinates, where $\left\{x^{i}\right\}$ is fixed in a neighbourhood of every point $x \in M$. We shall adopt Einstein summation convention with repeated indices summed up. The Riemannian metric $g(x)$ at any point $x \in M$ is a bilinear symmetric positive definite matrix denoted in local coordinates by $g_{i j}(x)=g_{i j} d x^{i} d x^{j}$. The Riemann structure allows us to define Riemannian volume measure $d \mu_{g}$ on $M$ by $d \mu_{g}=\sqrt{\left|g_{i j}(x)\right|} d x^{i}$. By the divergence theorem, we have the following integration by parts formulas: Let $X$ be a vector field, $X=X^{i} \partial_{i}$ and $f \in C^{\infty}(M)$ be smooth function. Then

$$
\int_{M}\langle-\operatorname{div} X, f\rangle_{g}=\int_{M}\langle X, \nabla f\rangle_{g}=-\int_{M} \frac{1}{\sqrt{\operatorname{det} g}} f \partial_{i}\left(X^{i} \sqrt{\operatorname{det} g}\right) \sqrt{\operatorname{det} g} d x^{i}
$$

Also for functions $f, h \in C^{2}(M)$,

$$
\int_{M} f \Delta_{g} h d \mu=-\int_{M}\langle\nabla f, \nabla h\rangle_{g} d \mu=\int_{M} \Delta_{g} f h d \mu .
$$

In local coordinates, gradient, Hessian and covariant derivative are written as $\nabla f=f_{i}, \quad \nabla \nabla f=\nabla_{i} \nabla_{j} f=$ $f_{i j}$ and $\frac{\partial}{\partial x^{i}}=\partial_{i}$ respectively. Time derivative is written as $\frac{\partial}{\partial t} f=\partial_{t} f=f_{t}$ and then $f_{t, j}=\nabla\left(f_{t}\right)$. Also we have the metric norm $|\nabla f|_{g}^{2}=g^{i j} \nabla_{i} f \nabla_{j} f=\nabla^{j} f \nabla_{j} f$.

2.2. Evolution equations. Interestingly, all the geometric quantities associated with the underlying manifold evolve as the Riemannian metric evolves along the geometric flow. This also serves as a motivation considering the behaviours of some other important geometric quantities such as eigenvalues of the manifold under the flow. The next lemma give us these evolutions.

Lemma 2.1. Suppose a one-parameter family of smooth metrics $g(t)$ solves the geometric flow (1.1). Then, we have the following evolutions

$$
\begin{aligned}
& \frac{\partial}{\partial t} g^{i j}=2 g^{i k} g^{j l} h_{k l}=2 h^{i j} \\
& \frac{\partial}{\partial t} d \mu=-\mathscr{H} d \mu . \\
& \frac{\partial}{\partial t}|\nabla f|^{p}=p|\nabla f|^{p-2}\left\{h_{i j} \nabla_{i} f \nabla_{j} f+g^{i j} \nabla_{i} f \nabla_{j} f_{t}\right\} \\
& \frac{\partial}{\partial t}|\nabla f|^{p-2}=(p-2)|\nabla f|^{p-4}\left\{h_{i j} \nabla_{i} f \nabla_{j} f+g^{i j} \nabla_{i} f \nabla_{j} f_{t}\right\} \\
& \begin{array}{r}
\frac{\partial}{\partial t}\left(\Delta_{p, g} f\right)=2 h^{i j} \nabla_{i}\left(Z \nabla_{j} f\right)+g^{i j} \nabla_{i}\left(Z_{t} \nabla_{j} f\right)+g^{i j} \nabla_{i}\left(Z \nabla_{j} f_{t}\right) \\
\quad+Z\left\{2\langle\operatorname{div} h, \nabla f\rangle-\langle\nabla \mathscr{H}, \nabla f\rangle+\Delta f_{t}\right\},
\end{array}
\end{aligned}
$$

where $\mathscr{H}=g^{i j} h_{i j}$, the metric trace of a symmetric 2-tensor $h_{i j}$, div is the divergence operator i.e. $(\text { divh })_{k}=g^{i j} \nabla_{i} h_{j k}, Z:=|\nabla f|^{p-2}$ and $f$ is a smooth function defined on $M$.

The proof follows standard computation as in [9, Lemma 2.2], see [10, Lemmas 2.1 and 2.2] for the proof. 
2.3. Main Results. Recall that we already mentioned that sign assumption on tensor $h_{i j}$ is preserved throughout the flow. To prove the monotonicity of $\lambda_{p, 1}$, we will need the condition

$$
\left(h_{i j}-\alpha \mathscr{H} g_{i j}\right)(x, t) \geq 0, \quad \forall t \in[0, T] .
$$

This condition is informed by the Hamilton's maximum principle for tensors. For clarity we state the principle without proof.

Definition 2.2. (Null-eigenvector assumption [23]) A quantity $Q(q, t): S y m^{2} T^{*} M \times[0, T] \rightarrow S y m^{2} T^{*} M$ is said to satisfy the null eigenvector assumption if whenever $\omega_{i j}$ is a nonnegative symetric $(0,2)$-tensor at a point $q$ and if $X \in T_{q} M$ is such that $\omega_{i j} X^{j}=0$ and then

$$
Q_{i j}(\omega, g) X^{i} X^{j} \geq 0
$$

for any $t \in[0, T)$.

The symetric tensor $\omega_{i j}$ is defined to be nonnegative if and only if $\omega_{i j} V^{i} V^{j} \geq 0$ for all vectors $V^{i}$ (i.e., the quadratic form induced by $\omega_{i j}$ is semi-positive definite) and we write $\omega_{i j} \geq 0$.

Theorem 2.3. ([24, Theorem 9.1], [23, Theorem 4.6]) Let $g(t)$ be a smooth one parameter family of Riemannian metrics satisfying (1.1). Let $M_{i j}$ be a symmetric $(0,2)$-tensor satisfying

$$
\frac{\partial}{\partial t} M_{i j}(x, t) \geq \Delta M_{i j}(x, t)+\left\langle X, \nabla M_{i j}\right\rangle+Q\left(M_{i j}, g(t)\right),
$$

where $X$ is a time-dependent vector field and $Q\left(M_{i j}, g(t)\right)$ is a symmetric $(0,2)$-tensor which is locally Lipschitz in $x$, continuous in $t$ and satisfies the null-eigenvector assumption. If $M_{i j}(p, 0) \geq 0$ for all $p \in M$, then $M_{i j}(p, t) \geq 0$ for all $p \in M$ and $t \in\left[0, T_{\varepsilon}\right)$

This theorem is a version of the maximum principle for tensors, which was originally applied to the evolution of Ricci tensor under the 3-dimensional Ricci flow. In general. the condition of nonnegativity of the Ricci curvature tensor is not preserved under the Ricci flow for the dimension greater than 3 . However, by the above maximum principle for tensors we can prove the following proposition.

Proposition 2.4. Let $g(t)$ be a smooth one parameter family of Riemannian metrics satisfying (1.1). If

$$
\left(h_{i j}-\alpha \mathscr{H} g_{i j}\right)(x, 0) \geq 0,
$$

then

$$
\left(h_{i j}-\alpha \mathscr{H} g_{i j}\right)(x, t) \geq 0
$$

for some $\alpha \in\left[0, \frac{1}{n}\right]$ and all $t \in[0, T)$.

The proof of this proposition follows directly from the proof of [24, Theorem 9.4] (see also [21, Lemma 4.3]) by applying Theorem 2.3 with $M_{i j}=h_{i j}-\alpha \mathscr{H} g_{i j}$. Hence, we omit it here. The only technicality involved is to get the time evolution of $(0,2)$-tensor $h_{i j}$ under the flow (1.1).

The main results can now be stated, whereas the proofs will be discussed in Section 3.

Theorem 2.5. Let $(M, g)$ be an n-dimensional closed Riemannian manifold evolving by geometric flow (1.1). Let $\lambda_{p, 1}(t)$ be the first eigenvalue of the p-Laplacian on $M$ corresponding to the eigenfunction $u(t, x)$ at time $t \in[0, T]$. Then

$$
\frac{d}{d t} \lambda_{p, 1}(t)=\lambda_{p, 1}(t) \int_{M} \mathscr{H}|u|^{p} d \mu-\int_{M} \mathscr{H}|\nabla u|^{p} d \mu+p \int_{M}|\nabla u|^{p-2} h^{i j} \nabla_{i} u \nabla_{j} u d \mu
$$


for all time $t \in[0, T]$. Moreover, if

$$
h_{i j}-\alpha \mathscr{H} g_{i j} \geq 0, \quad \alpha \in[1 / p, 1 / n) \text {, }
$$

then

$$
\frac{d}{d t} \lambda_{p, 1}(t) \geq \lambda_{p, 1}(t) \int_{M} \mathscr{H}|u|^{p} d \mu+(\alpha p-1) \int_{M} \mathscr{H}|\nabla u|^{p} d \mu \geq 0
$$

provided $\mathscr{H}$ is nonnegative.

Corollary 2.6. Under the same assumptions of Theorem 2.5.

$$
\lambda_{p, 1}\left(t_{2}\right) \geq \lambda_{p, 1}\left(t_{1}\right)+\int_{t_{1}}^{t_{2}} \Theta(g(t), u(t)) d t
$$

where

$$
\Theta(g(t), u(t))=\lambda_{p, 1}(t) \int_{M} \mathscr{H}|u|^{p} d \mu+(\alpha p-1) \int_{M} \mathscr{H}|\nabla u|^{p} d \mu
$$

for $t_{1}, t_{2} \in[0, T], T<\infty$. Furthermore, if $\mathscr{H} \geq \mathscr{H}_{\min } \geq 0$, then, $\forall t_{1}<t_{2}$,

$$
\lambda_{p, 1}\left(t_{2}\right) \geq \lambda_{p, 1}\left(t_{1}\right) \exp \left\{\alpha p \int_{t_{1}}^{t_{2}} \mathscr{H}_{\min }(t) d t\right\}
$$

and $\lambda_{p, 1}(t)$ is monotonically nondecreasing along flow.

Finally, we obtain a monotonic quantity involving $\lambda_{p, 1}(t)$ and then show that $\lambda_{p, 1}(t)$ is differentiable almost everywhere along the geometric flow.

Theorem 2.7. Under the same assumptions of Theorem 2.5, if instead $\mathscr{H} \geq \mathscr{H}_{\min }(0) \neq 0$ (i.e., either $\mathscr{H}_{\text {min }}(0)>0$ or $\left.\mathscr{H}_{\min }(0)<0\right)$, then the following quantity $\lambda_{p, 1}(t) \cdot\left(\mathscr{H}_{\min }(0)^{-1}-\frac{2}{n} t\right)^{\frac{\alpha n p}{2}}$ is monotonically nondecreasing and $\lambda_{p, 1}(t)$ is differentiable almost everywhere along the geometric flow (1.1).

\section{VARIATION AND MONOTONICITY FORMULAS}

Now consider the eigenvalue problem

$$
\Delta_{p, g} u=-\lambda|u|^{p-2} u, \quad u \neq 0 \quad \text { on } M \times[0, T]
$$

with the normalisation condition $\int_{M}|u|^{p} d \mu=1$. We want to derive general evolution for the eigenvalues of $\Delta_{p, g}$ and show that $\lambda_{p, 1}$ is monotone on metrics evolving by the geometric flow. In order to do these, we need to calculate time evolution for $\lambda_{p, 1}$ and its corresponding eigenfunction. To avoid differentiability assumption on $\lambda_{p, 1}$ or its corresponding eigenfunction along the flow, we apply some techniques similar to the one in [8] as discussed above. Precisely, let $(M, g(t)), t \in[0, T]$ be a smooth closed Riemannian manifold evolving by the flow (1.1). Define a general smooth function as follows

$$
\lambda_{p, 1}(u(t), t):=-\int_{M} u(t) \Delta_{p} u(t) d \mu_{g(t)}=\int_{M}|\nabla u(t)|^{p} d \mu_{g(t)},
$$

where $u(t)$ is a smooth function satisfying the normalisation condition

$$
\int_{M}|u(t)|^{p} d \mu_{g(t)}=1 \text { and } \int_{M}|u(t)|^{p-2} u(t) d \mu_{g(t)}=0 .
$$


By this we claim that there exists a smooth function $u(s)$ at time $t=s \in[0, T]$ satisfying (3.3). To see this claim, we first assume that, at time $t=s, u(s)$ is the eigenfunction corresponding to $\lambda_{p, 1}(s)$ of $\Delta_{p . g(s)}$, which implies

$$
\int_{M}|u(s)|^{p} d \mu_{g(s)}=1 \text { and } \int_{M}|u(s)|^{p-2} u(s) d \mu_{g(s)}=0 .
$$

Then we consider the following smooth function

$$
f(t)=u(s)\left(\frac{|g(s)|}{|g(t)|}\right)^{\frac{1}{2(p-2)}}
$$

under the flow $g(t)$. We normalize this smooth function

$$
u(t)=\frac{f(t)}{\left(\int_{M}|f(t)|^{p} d \mu_{g(t)}\right)^{\frac{1}{p}}}
$$

under the flow $g(t)$. By (3.5), we can easily check that $u(t)$ satisfies (3.3). In general, $\lambda_{p, 1}(u, t)$ is not equal to $\lambda_{p, 1}(t)$. But at time $t=s$, if $u(s)$ is the eigenfunction of the first eigenvalue $\lambda_{p, 1}(s)$, then

$$
\lambda_{p, 1}(u(s), s)=\lambda_{p, 1}(s)
$$

and

$$
\frac{d}{d t} \lambda_{p, 1}(u(t), t)=\frac{d}{d t} \lambda_{p, 1}(t)
$$

at some time $t=s$. Notice that the normalisation condition implies

$$
\left.\frac{\partial}{\partial t}\left(\int_{M}|u|^{p} d \mu\right)\right|_{t=s}=0
$$

which by direct computation (at $t=s$ ) yields the following

$$
\int_{M}|u|^{p-2} u\left((p-1) \frac{\partial u}{\partial t} d \mu+\frac{\partial}{\partial t}(u d \mu)\right)=0 .
$$

We are now set to prove a theorem about the evolution, monotonicity and differentiability (Theorem 2.5 ) of the first eigenvalue of the $p$-Laplacian under the geometric flow. Clearly, we can now set

$$
\lambda_{p, 1}(t)=\lambda_{p, 1}(u(t), t)=-\int_{M} u(t, x) \Delta_{p} u(t, x) d \mu_{g(t)} .
$$

The evolution of $\lambda_{p, 1}$ then follows

$$
\frac{d}{d t} \lambda_{p, 1}(t)=\left.\frac{\partial}{\partial t} \lambda_{p, 1}(u(t), t)\right|_{t=s}=-\frac{\partial}{\partial t} \int_{M} u(t, x) \Delta_{p} u(t, x) d \mu_{g(t)} .
$$

\section{Proof of Theorem 2.5.}


Proof. The proof also follows by direct computation using evolution of quantities in Lemma 2.1. Denote $Z:=|\nabla u|^{p-2}$. It follows that

$$
\begin{aligned}
\frac{\partial}{\partial t} \int_{M} u \Delta_{p} u d \mu & =\frac{\partial}{\partial t} \int_{M} g^{i j} \nabla_{i}\left[Z \nabla_{j} u\right] u d \mu \\
& =\frac{\partial}{\partial t} \int_{M}\left(g^{i j} \nabla_{i} Z \nabla_{j} u+Z \Delta u\right) u d \mu \\
& =\int_{M} \frac{\partial}{\partial t}\left(g^{i j} \nabla_{i} Z \nabla_{j} u+Z \Delta u\right) u d \mu+\int_{M} \Delta_{p} u \frac{\partial}{\partial t}(u d \mu) \\
& =: I+I I .
\end{aligned}
$$

By the evolution of $\Delta_{p, g}$ in Lemma 2.1, we have

$$
I=\int_{M} 2 h^{i j} \nabla_{i}\left(Z \nabla_{j} u\right)+g^{i j} \nabla_{i}\left(Z_{t} \nabla_{j} u\right)+g^{i j} \nabla_{i}\left(Z \nabla_{j} u_{t}\right)+\int_{M} Z\{2\langle\operatorname{div} h, \nabla u\rangle-\langle\nabla \mathscr{H}, \nabla u\rangle\} u d \mu .
$$

Using integration by parts on the second and third terms of the last integral, we have

$$
\left.I=\int_{M} 2 h^{i j} \nabla_{i}\left(Z \nabla_{j} u\right)-\int_{M} g^{i j} Z_{t} \nabla_{i} u \nabla_{j} u-\int_{M} Z g^{i j} \nabla_{i} u \nabla_{j} u_{t}\right)+\int_{M} Z\{2\langle\operatorname{div} h, \nabla u\rangle-\langle\nabla \mathscr{H}, \nabla u\rangle\} u d \mu .
$$

Therefore we have after using the evolution $Z_{t}$ from Lemma 2.1 that

$$
\begin{array}{rl}
\frac{\partial}{\partial t} \int_{M} u \Delta_{p} u d \mu=\int_{M} & 2 h^{i j} \nabla_{i}\left(Z \nabla_{j} u\right) u d \mu-(p-2) \int_{M}|\nabla u|^{p-2} h^{i j} \nabla_{i} u \nabla_{j} u d \mu \\
& -(p-1) \int_{M}|\nabla u|^{p-1} g^{i j} \nabla_{i} u \nabla_{j} u_{t} d \mu \\
& +\int_{M} Z\{2\langle\operatorname{div} h, \nabla u\rangle-\langle\nabla \mathscr{H}, \nabla u\rangle\} u d \mu \\
& +\int_{M} \Delta_{p} u \frac{\partial}{\partial t}(u d \mu) .
\end{array}
$$

Computing the first and the third terms on the right hand side of (3.10) as follows

$$
\begin{aligned}
\int_{M} 2 h^{i j} \nabla_{i}\left(Z \nabla_{j} u\right) u d \mu & =-2 \int_{M} \nabla_{i}\left(h^{i j} u\right) Z \nabla_{j} u u d \mu \\
& =-2 \int_{M}|\nabla u|^{p-2} h^{i j} \nabla_{i} u \nabla_{j} u d \mu-2 \int_{M} Z\langle d i v h, \nabla u\rangle u d \mu . \\
-(p-1) \int_{M}|\nabla u|^{p-1} g^{i j} \nabla_{i} u \nabla_{j} u_{t} d \mu & =(p-1) \int_{M} g^{i j} \nabla_{i}\left(|\nabla u|^{p-1} \nabla_{i} u\right) u_{t} \\
& =(p-1) \int_{M} \Delta_{p} u u_{t} d \mu
\end{aligned}
$$

Putting these back into (3.10), we have

$$
\begin{aligned}
\frac{\partial}{\partial t} \int_{M} u \Delta_{p} u d \mu= & -p \int_{M}|\nabla u|^{p-2} h^{i j} \nabla_{i} u \nabla_{j} u d \mu-\int_{M} Z\langle\nabla \mathscr{H}, \nabla u\rangle u d \mu \\
& +(p-1) \int_{M} \Delta_{p} u u_{t} d \mu+\int_{M} \Delta_{p} u \frac{\partial}{\partial t}(u d \mu) .
\end{aligned}
$$

Using the normalisation condition (3.7) and the definition of $\Delta_{p} u$ in (3.1), the last two terms on the RHS of the above equation vanish and we then arrive at

$$
\frac{\partial}{\partial t} \int_{M} u \Delta_{p} u d \mu=-p \int_{M}|\nabla u|^{p-2} h^{i j} \nabla_{i} u \nabla_{j} u d \mu-\int_{M} Z\langle\nabla \mathscr{H}, \nabla u\rangle u d \mu .
$$


The next is to compute the second term on the RHS of the last equality using integration by parts as follows

$$
\begin{aligned}
-\int_{M} Z\langle\nabla \mathscr{H}, \nabla u\rangle u d \mu & =\int_{M} \mathscr{H} \nabla_{i}\left(Z \nabla_{j} u u\right) d \mu \\
& =\int_{M} \mathscr{H} \nabla_{i}\left(Z \nabla_{j} u\right) u d \mu+\int_{M} \mathscr{H} Z \nabla_{i} u \nabla_{j} u d \mu \\
& =\int_{M} \mathscr{H} \Delta_{p} u u d \mu+\int_{M} \mathscr{H}|\nabla u|^{p-2}|\nabla u|^{2} d \mu \\
& =-\lambda_{p, 1}(t) \int_{M} \mathscr{H}|u|^{p} d \mu+\int_{M} \mathscr{H}|\nabla u|^{p} d \mu
\end{aligned}
$$

Putting this into (3.11), we obtain (2.3) at once. Hence, the first part of the theorem is proved. Using the condition (2.4) in (2.3), we have the monotonicity formula (2.5) with the condition $\mathscr{H} \geq 0$.

Remark 3.1. Clearly, when $p=2$, we have $\Delta_{2}=\Delta_{g}$, the usual Laplace-Beltrami operator. Also $\lambda_{p, 1}=$ $\lambda_{1}$, the first eigenvalue of $\Delta_{g}$ and the corresponding eigenfunction are smoothly differentiable. Then, Theorem [10, Theorem 2.6] reduces to a corollary. This further explains that the $p$-Laplacian is a nonlinear generalisation of Laplace-Beltrami operator. Integrating both sides of (2.5) from $t_{1}$ to $t_{2}$ on a sufficiently small time interval $t_{1} \leq t \leq t_{2}, t \in[0, T]$, we then obtain 2.6.

Proof of Corollary 2.6. Using (2.5) we have

$$
\frac{d}{d t} \lambda_{p, 1}(t) \geq \Theta(g(t), u(t))
$$

where

$$
\Theta(g(t), u(t))=\lambda_{p, 1}(t) \int_{M} \mathscr{H}|u|^{p} d \mu+(\alpha p-1) \int_{M} \mathscr{H}|\nabla u|^{p} d \mu .
$$

Integrating (3.12) from $t_{1}$ to $t_{2}, t_{1}, t_{2} \in[0, T], T<\infty$ yields

$$
\lambda_{p, 1}\left(t_{2}\right)-\lambda_{p, 1}\left(t_{1}\right) \geq \int_{t_{1}}^{t_{2}} \Theta(g(t), u(t)) d t
$$

Now, let $\mathscr{H} \geq \mathscr{H}_{\text {min }}$. By the definition of $\lambda_{p, 1}$ in (1.3) and the normalisation condition $\int_{M}|u|^{p} d \mu=1$, we know that $\lambda_{p, 1}=\int_{M}|\nabla u|^{p} d \mu$ Hence (2.5) is reduced to

$$
\frac{d}{d t} \lambda_{p, 1}(t) \geq \alpha p \mathscr{H}_{\min }(t) \lambda_{p, 1}(t)
$$

from which (2.7) follows by integrating on the interval $\left[t_{1}, t_{2}\right]$ with $t_{1}, t_{2} \in[0, T]$. This implies that $\lambda_{p, 1}(t)$ is monotonically nondecreasing in the interval $\left[t_{1}, t_{2}\right]$.

Proof of Theorem 2.7. Note that both $\lambda_{1}(t)$ and $\mathscr{H}_{\min }(t)$ are functions of time only. Setting $\mathscr{H}_{\min }(0)=\psi_{0}$, we can evaluate (See (4.5) in the Appendix)

$$
\begin{aligned}
\int_{t_{1}}^{t_{2}} \mathscr{H}_{\min }(t) d t & =\int_{t_{1}}^{t_{2}}\left(\frac{1}{\psi_{0}^{-1}-\frac{2}{n} t}\right) d t \\
& =-\left.\frac{n}{2} \log \left(\psi_{0}^{-1}-\frac{2}{n} t\right)\right|_{t_{1}} ^{t_{2}}=\log \left(\frac{\psi_{0}^{-1}-\frac{2}{n} t_{1}}{\psi_{0}^{-1}-\frac{2}{n} t_{2}}\right)^{\frac{n}{2}} .
\end{aligned}
$$

Therefore integrating both sides of (3.13) from $t_{1}$ to $t_{2}$ and together with the above equality yields that

$$
\log \frac{\lambda_{p, 1}\left(t_{2}\right)}{\lambda_{p, 1}\left(t_{1}\right)} \geq \log \left(\frac{\psi_{0}^{-1}-\frac{2}{n} t_{1}}{\psi_{0}^{-1}-\frac{2}{n} t_{2}}\right)^{\frac{\alpha n p}{2}}
$$


for any time $t_{1}<t_{2}$ sufficiently close to $t_{2}$. Hence,

$$
\lambda_{p, 1}\left(t_{2}\right) \cdot\left(\psi_{0}^{-1}-\frac{2}{n} t_{2}\right)^{\frac{\alpha n p}{2}} \geq \lambda_{p, 1}\left(t_{1}\right) \cdot\left(\psi_{0}^{-1}-\frac{2}{n} t_{1}\right)^{\frac{\alpha n p}{2}},
$$

which means that the quantity $\lambda_{p, 1}(t) \cdot\left(\psi_{0}^{-1}-\frac{2}{n} t\right)^{\frac{\alpha n p}{2}}$ in the interval $\left[t_{1}, t_{2}\right]$ along the geometric flow. Notice that $\left(\psi_{0}^{-1}-\frac{2}{n} t\right)$ is decreasing in the interval $t_{1}<t_{2}, t_{1}, t_{2} \in[0, T)$. This means that $\lambda_{p, 1}(t)$ is nondecreasing along the flow.

The differentiability of $\lambda_{p, 1}(t)$. Since $\lambda_{p, 1}(t)$ is nondecreasing on the time interval $[0, T)$ (under curvature assumption of the theorem), by the classical Lebesgue's theorem [25, Chapter 4], it is easy to see that $\lambda_{p, 1}(t)$ is differentiable almost everywhere.

Remark 3.2. The proofs of the first eigenvalue variation and monotonicity do not use any differentiability of the first eigenvalue $\lambda_{p, 1}(t)$ or its corresponding eigenfunction $u(t, x)$ of the $p$-Laplacian under the flow. In fact, it is not known whether they are differentiable in advance. It would be interesting to find out whether the corresponding first eigenfunction of the $p$-Laplacian is a $C^{1}$-differentiable function with respect to $t$-variable along the flow.

\section{APPENDIX}

A. Geomtric flow. The monotonicity of $\lambda_{p, 1}(t)$ depends on the sign of $\mathscr{H}$. Note that in applications the sign of $\mathscr{H}$ is usually preserved throughout the evolution. An interesting case is when the manifold is being evolved under the Ricci flow [24], where the nonnegativity of scalar curvature is preserved. Notice also that $\mathscr{H}$ evolves by

$$
\frac{\partial}{\partial t} \mathscr{H}=\beta+2\left|h_{i j}\right|^{2}
$$

where $\beta:=g^{i j} \partial_{t} h_{i j}$. In particular, under Ricci flow where $h_{i j}=R_{i j}$ and $\mathscr{H}=R$, we have $\beta=\Delta R$. Here we will assume that

$$
\beta-\Delta \mathscr{H} \geq 0
$$

This is motivated by an error term appearing in a result of Müller [26, Lemma 1.6]. For our case the error term reads; for any time-dependent vector field $X$ on $M$

$$
\mathscr{D}(X):=2\left(R_{i j}-h_{i j}\right)(X, X)+2\langle 2 \operatorname{div} h-\nabla \mathscr{H}, X\rangle+\partial_{t} \mathscr{H}-\Delta \mathscr{H}-2\left|h_{i j}\right|^{2},
$$

where $R_{i j}$ is the Ricci curvature tensor of $M$. Clearly the last three terms in (4.2) above is the same as the quantity $\beta-\Delta \mathscr{H}$. It does make sense to assume (4.1) holds whenever $\mathscr{D}(X)$ is nonnegative. An application of this is that the flow is a steady or shrinking soliton (self-similar solution to the geometric flow) if the equality in (4.1) holds. Writing $\left|h_{i j}\right|^{2} \geq \frac{1}{n} \mathscr{H}^{2}$ and using the condition that $\beta-\Delta \mathscr{H} \geq 0$, we have that a governing differential inequality for the evolution of $\mathscr{H}$ follows

$$
\frac{\partial}{\partial t} \mathscr{H} \geq \Delta \mathscr{H}+\frac{2}{n} \mathscr{H}^{2}
$$

Supposing $\mathscr{H} \geq \mathscr{H}_{\min }$, we can apply the maximum principle by comparing the solution of (4.3) with that of the following ordinary differential equation

$$
\left\{\begin{aligned}
\frac{d \psi(t)}{d t} & =\frac{2}{n}(\psi(t))^{2} \\
\psi(0) & =\mathscr{H}_{\min }(0)
\end{aligned}\right.
$$


Therefore

$$
\mathscr{H}_{g(t)} \geq \psi(t)=\frac{\mathscr{H}_{\min }(0)}{1-\frac{2}{n} \mathscr{H}_{\min }(0) t}
$$

for all $t \geq 0$ as long as the flow exists.

B. Examples of geometric flows. In this section, we give some examples of geometric flows where our results are valid. We remark that in these cases the error term $\mathscr{D}(X)$ and the quantity $\beta-\Delta \mathscr{H}$ are nonnegative. More examples can be found in [26, Section 2].

B.1. Hamilton's Ricci flow [24]. Let $(M, g(t))$ be a solution to the Hamilton's Ricci flow

$$
\partial_{t} g_{i j}(t, x)=-2 R_{i j}
$$

This is the case where $h_{i j}=R_{i j}$ is the Ricci tensor and $\mathscr{H}=R$ is the scalar curvature on $M$. Here, the scalar curvature evolves by

$$
\partial_{t} R=\Delta R+2\left|R_{i j}\right|^{2}
$$

By twice contracted second Bianchi identity $g^{i j} \nabla_{i} R_{j k}=\frac{1}{2} \nabla_{k} R$, which implies

$$
2\langle\operatorname{div} h, \nabla f\rangle-\langle\nabla \mathscr{H}, \nabla f\rangle=0,
$$

the quantity $\mathscr{D}(X)$ vanishes identically and $\beta-\Delta R \equiv 0$. Note that the positivity of curvature is preserved along the Ricci flow [24]. The evolution equation and monotonicity formula for the first eigenvalue follow easily (see [8, 12, 27] and [7, 13, 14]). A fundamental result here is Perelman's paper [28], where he defines his $\mathscr{F}$-energy

$$
\mathscr{F}\left(g_{i j}(t), u(t)\right)=\int_{M}\left(4|\nabla u|^{2}+R u^{2}\right) d \mu \quad \text { with } \quad \int_{M} u^{2} d \mu=1 .
$$

and proved that it is monotonically nondecreasing. He also defined

$$
\lambda_{1}\left(g_{i j}\right)=\inf \left\{\mathscr{F}\left(g_{i j}, f\right): f \in C_{c}^{\infty}(M), \int_{M} e^{-f} d \mu=1\right\},
$$

with $\lambda_{1}\left(g_{i j}\right)$ (being the least eigenvalue of the geometric operator $\left.-4 \Delta+R\right)$ and its corresponding eigenfunction $u=e^{-f}$ satisfying the eigenvalue problem

$$
-4 \Delta u+R u=\lambda_{1}\left(g_{i j}\right) u .
$$

He showed that monotonicity of $\lambda_{1}\left(g_{i j}\right)$ follows from that of $\mathscr{F}$.

B.2 Ricci harmonic flow [29]. Let $(M, g)$ and $(N, \xi)$ be compact (without boundary) Riemannian manifolds of dimensions $m$ and $n$ respectively. Let a smooth map $\varphi: M \rightarrow N$ be a critical point of the Dirichlet energy integral $E(\varphi)=\int_{M}|\nabla \varphi|^{2} d \mu_{g}$, where $N$ is isometrically embedded in $\mathbb{R}^{d}, d \geq n$, by the Nash embedding theorem. The configuration $(g(x, t), \varphi(x, t)), t \in[0, T)$ of a one parameter family of Riemannian metrics $g(x, t)$ and a family of smooth maps $\varphi(x, t)$ is defined to be Ricci-harmonic map flow if it satisfies the coupled system of nonlinear parabolic equations

$$
\begin{aligned}
& \frac{\partial}{\partial t} g(x, t)=-2 R c(x, t)+2 \alpha \nabla \varphi(x, t) \otimes \nabla \varphi(x, t), \\
& \frac{\partial}{\partial t} \varphi(x, t)=\tau_{g} \varphi(x, t),
\end{aligned}
$$


where $R c(x, t)$ is the Ricci curvature tensor for the metric $g, \alpha(t) \equiv \alpha>0$ is a time-dependent coupling constant, $\tau_{g} \varphi$ is the intrinsic Laplacian of $\varphi$, which denotes the tension field of map $\varphi$ and $\nabla \varphi \otimes \nabla \varphi=$ $\varphi^{*} \xi$ is the pullback of the metric $\xi$ on $N$ via the map $\varphi$. Here $h_{i j}=R_{i j}-\alpha \partial_{i} \varphi \partial_{j} \varphi=: S_{i j}, \quad \mathscr{H}=$ $R-\alpha|\nabla \varphi|^{2}=: S$ and

$$
\partial_{t} S=\Delta S+2\left|S_{i j}\right|^{2}+2 \alpha\left|\tau_{g} \varphi\right|^{2}-2 \dot{\alpha}|\nabla \varphi|^{2} .
$$

Using the twice contracted second Bianchi identity, we have

$$
\left(g^{i j} \nabla_{i} S_{j k}-\frac{1}{2} \nabla_{k} S\right) X_{j}=-\alpha \tau_{g} \varphi \nabla_{j} \varphi X_{j}
$$

A straightforward computation gives

$$
2(R c-\mathscr{S})(\nabla u, \nabla u)=2 \alpha|\nabla \varphi|^{2}|\nabla u|^{2} .
$$

Then,

$$
\mathscr{D}(X)=2 \alpha\left|\tau_{g} \varphi-\langle\nabla \varphi, X\rangle\right|^{2}-2 \dot{\alpha}|\nabla \varphi|^{2} \text { and } \beta-\Delta S=2 \alpha\left|\tau_{g} \varphi\right|^{2}-\dot{\alpha}|\nabla \varphi|^{2}
$$

for all $X$ on $M$. Thus, both $\mathscr{D}$ and $\beta-\Delta S$ are nonnegative as long as $\alpha(t)$ is nonincreasing in time. The first author has considered this in [9]. See also [17] for the monotonicity of the first eigenvalue of Laplace-Betrami operator and versions of Perelman's entropy under the Ricci-harmonic map flow.

B.3. Lorentzian mean curvature flow. Let $M^{n}(t) \subset L^{n+1}$ be a family of space-like hypersurfaces in ambient Lorentzian manifold evolving by Lorentzian mean curvature flow

$$
\partial_{t} F(t, \cdot)=\Pi(t, \cdot) v(t, \cdot)
$$

for $(t, \cdot) \in[0, T] \times M$, where $F(t, \cdot)$ is the position of $M^{n}$ in $L^{n+1}$ satisfying $F(0, \cdot)=F_{0}(\cdot)$. Here $v(t, \cdot)$ and $\Pi(t, \cdot)$ are respectively the outer normal vector and mean curvature at the point $F(t, \cdot)$. Then, the induced metric evolves by

$$
\partial_{t} g_{i j}=2 H \Pi_{i j}
$$

where $\Pi_{i j}$ denotes the components of the second fundamental form $\Pi$ on $M$ and $H=g^{i j} \Pi_{i j}$ denotes the mean curvature of $M$. In this case $h_{i j}=-H \Pi_{i j}$ and $\mathscr{H}=-H^{2}$. Letting $\widetilde{R c}$ and $\widetilde{R m}$ denote the Ricci and Riemman curvature tensor of $L^{n+1}$ respectively, we have the Gauss equation

$$
R_{i j}=\widetilde{R}_{i j}-H \Pi_{i j}+\Pi_{i l} \Pi_{l j}+\widetilde{R}_{i 0 j 0},
$$

the Codazzzi equation

$$
\nabla_{i} \Pi_{j k}-\nabla_{k} \Pi_{i j}=\widetilde{R}_{0 j k i}
$$

the evolution equation

$$
\partial_{t} H=\Delta H-H\left(|\Pi|^{2}+\widetilde{R c}(v, v)\right.
$$

and

$$
\beta-\Delta H=2 H^{2}|\Pi|^{2}+|\nabla H|^{2}+2 H \widetilde{R c}(v, v) .
$$

See the explicit forms of the Gauss and the Codazzi equations for $L^{n+1}=\mathbb{R}^{n+1}$ in [30]. Combining the above equation we obtain the quantity

$$
\mathscr{D}(X)=2|\nabla H-\Pi(X, \cdot)|^{2}+2 \widetilde{R c}(H v-X, H v-X)+2\langle\widetilde{R m}(X, v) v, X\rangle,
$$

where $v$ denotes future-oriented timelike unit normal vector on $M$. Obviously both $\mathscr{D}(X)$ and $\beta-\Delta H$ are nonnegative when assuming nonnegativity on sectional curvature of $L^{n+1}$. See [22] for the evolution and 
monotonicity of the first eigenvalue of $p$-Laplace operator under the $m^{\text {th }}$ powers of the mean curvature flow. See also Huisken [30], the last author's paper [21] and [20, 22] for related results.

B.4. The Yamabe flow. This is the case when $h_{i j}=\frac{1}{2} R g_{i j}$, where $R$ is the scalar curvature of the metric. Yamabe flow is then the following evolution equation

$$
\begin{aligned}
& \frac{\partial}{\partial t} g_{i j}(x, t)=-R(x, t) g_{i j}(x, t), \quad(x, t) \in M \times[0, T], \\
& g_{i j}(x, 0)=g_{0}(x)
\end{aligned}
$$

as introduced by Hamilton who first established the existence of its unique solution for all time and shows that the metric $g(t)$ approaches constant as $t \rightarrow \infty$. His proof was done for volume preserving flow

$$
\frac{\partial}{\partial t} g_{i j}(x, t)=(r(t)-R(x, t)) g_{i j}(x, t), \quad(x, t) \in M \times[0, \infty),
$$

with $r(t)=\operatorname{Vol}^{-1}(g(t)) \int_{M} R d \mu$ is the average of scalar curvature for the metric in a conformal class. For more on the global existence and convergence of (4.14) see Chow [31] and Ye [32]. Note that under the Yamabe flow the volume measure evolves as $\partial_{t} d \mu=n / 2 R d \mu$ and the normalization condition, $\partial_{t}\left(\int_{M}|u|^{p} d \mu\right)=0$, implies

$$
\int_{M} p|u|^{p} u u_{t} d \mu-\frac{n}{2} \int_{M} R u d \mu=0 .
$$

Here, the evolution of scalar curvature was given [31] as

$$
\frac{\partial}{\partial t} R=(n-1) \Delta R+R^{2}
$$

and by the strong maximum principle

$$
R(x, t) \geq \psi(t)=\frac{R_{\min }(0)}{1-R_{\min }(0) t}
$$

for all t. We can also compute

$$
\begin{aligned}
\langle 2 \operatorname{div} h-\nabla \mathscr{H}, \nabla f\rangle & =\left\langle 2 g^{i j} \nabla_{i}\left(\frac{1}{2} R g_{i j}\right)-\frac{n}{2} \nabla_{k} R, \nabla f\right\rangle \\
& =\frac{n}{2}\langle\nabla R, \nabla f\rangle
\end{aligned}
$$

and

$$
\beta-\Delta \mathscr{H}=\frac{n(n-2)}{2} \Delta R
$$

These imply that the quantitty $\mathscr{D}(\nabla f)$ is nonnegative on the Einstein tensor

$$
E_{i j}=R_{i j}-\frac{1}{2} R g_{i j} \geq 0 \text {. }
$$

Hence, the main results of this paper hold for Yamabe flow. Using the assumptions that $p \geq n$ and $R \geq 0$, the author in [11, Section 7] proved that $\lambda_{p, 1}$ is strictly increasing and differentiable almost everywhere along the Yamabe flow. Yamabe flow coincides with the Ricci flow on Riemann surfaces.

\section{Funding}

The first two authors were supported by Landmark University, Omu-Aran, Nigeria. The third author was partially supported by the NSF of China (Grant No. 11401131). 


\section{REFERENCES}

[1] L. C. Evans, A new proof of local $C^{1, \alpha}$-regularity for solutions of certain degenerate elliptic PDE, J. Differential Equations 45 (1982), 356-373.

[2] P. Tolksdorf, Regularity for a more general class of quasilinear elliptic equations, J. Differential Equations 5 (1984), 126-150.

[3] P. Lindqvist, On the equation $\operatorname{div}\left(|\nabla u|^{p-2} \nabla u\right)+\lambda|u|^{p-2} u=0$, Research reports, vol. A 263, Helsinki Univ. Tech. Inst. Math. Helsinki (1988).

[4] P. Lindqvist, Addendum: On the equation $\operatorname{div}\left(|\nabla u|^{p-2} \nabla u\right)+\lambda|u|^{p-2} u=0$, Proc. Amer. Math. Soc. 116 (1992), $583-584$.

[5] T. Kato, Perturbation Theory for Linear Operator, Springer, Berlin, New York, 1984.

[6] B. Kleiner, J. Lott, Note on Perelman's paper, Geometry and Topology, 12 (2008), 2587-2858.

[7] L. Ma, Eigenvalue monotonicity for the Ricci-Hamilton flow, Ann. Global Anal. Geom. 29 (2006), $287-292$.

[8] X. Cao, Eigenvalues of $\left(-\Delta+\frac{R}{2}\right)$ on manifolds with nonnegative curvature operator, Math. Ann. 337 (2007), $435-442$.

[9] A. Abolarinwa, Evolution and monotonicity of the first eigenvalue of $p$-Laplacian under the Ricci- harmonic flow, J. Appl. Anal. 21 (2015), 147-160.

[10] A. Abolarinwa, J. Mao, The first eigenvalue of the p-Laplacian on time dependent Riemannian metrics, arxiv.org 1604.05884, 2016.

[11] J. Wu, First eigenvalue monotonicity for the $p$-Laplace operator under the Ricci flow, Acta Math. Sinica 27 (2011), 1591-1598.

[12] X. Cao, First eigenvalues of geometric operators under the Ricci flow, Proc. Amer. Math. Soc. 136 (2008), $4075-4078$.

[13] J-F. Li, Eigenvalues and energy functionals with monotonicity formulae under Ricci flow, Math. Ann. 338 (2007), 927946.

[14] J. Li, Monotonicity formulae under rescaled Ricci flow, arXiv:math/07010.5328v3 [math.DG] (2007).

[15] A. Abolarinwa, Eigenvalues of Weighted-Laplacian under the extended Ricci flow, Adv. Geom. 19 (2019), $131-143$.

[16] A. Abolarinwa, O. Adebimpe, E. A. Bakare, Monotonicity formulas for the first eigenvalue of the weighted $p$-Laplacian under the Ricci-harmonic flow, J. Ineq. Appl. 2019 (2019), Article ID 10.

[17] Y. Li, Eigenvalues and entropies under the Harmonic-Ricci Flow, Pacific J. Math. 267 (2014), 141-184.

[18] H. Guo, R. Philipowski, A. Thalmaier, Entropy and lowest eigenvalue on evolving manifolds, Pacific J. Math. 264 (2013), 61-82.

[19] J. Mao, Eigenvalue inequalities for the $p$-Laplacian on Riemannian manifold and estimates for the heat kernel, J. Math. Pures Appl. 101 (2014), 372-393.

[20] F. Guo, G. Li, C. Wu, Isoperimetric inequalities for eigenvalues by inverse mean curvature, arXiv:1602.05290v1 [math.DG].

[21] J. Mao, Monotonicity of the first eigenvalue of the Laplace and the $p$-Laplace operators under a forced mean curvature flow, J. Korean Math. Soc. 55 (2018) 1435-1458.

[22] L. Zhao, The first eigenvalue of $p$-Laplace operator under mean curvature flow, China Ann. Math. 30 (2012), $539-544$.

[23] B. Chow and D. Knopf, The Ricci flow: An introduction, AMS, Providence, RI (2004)

[24] R. Hamilton, Three-manifolds with positive Ricci curvature, J. Differential Geom. 17 (1982), 253-306.

[25] A. Mukherjea, K. Pothoven, Real and Functional Analysis. Part A. Real Analysis (second edition), Plenum Press, New York, 1984.

[26] R. Müller, Monotone volume formulas for geometric flows, J. Reine Angew. Math. 643 (2010), 39-57.

[27] A. Abolarinwa, Gradient estimates for a nonlinear parabolic equation with potential under geometric flow, Electron. J. Differential Equations 2015 (2015), 1-11.

[28] G. Perelman, The entropy formula for the Ricci flow and its geometric application, arXiv:math.DG/0211159v1 (2002).

[29] R. Müller, Ricci flow coupled with harmonic map flow, Ann. Sci. Ec. Norm. Sup. 4 (2012),101-142.

[30] G. Huisken, Flow by mean curvature of convex surfaces into spheres, J. Differential Geom. 20 (1984) 237-266.

[31] B. Chow, The Yamabe flow on locally conformally flat manifolds with positive Ricci curvature, Commun. Pure. Appl. Math. 45 (1992), 1003-1014.

[32] R. Ye, Global existence and convergence of Yamabe flow, J. Differential Geom. 39 (1994), 35-50. 